\title{
Avaliação da Demanda Preliminar de Atendimento Dirigida pelo Aluno ao Núcleo de Apoio Psicopedagógico ao Estudante da Faculdade de Medicina (Napem) da Universidade Federal de Minas Gerais
}

\author{
Evaluation of the Student's Preliminary \\ Request for Assistance to the School of \\ Medicine Psychopedagogical Student Support \\ Center (NAPEM) of the Federal University of \\ Minas Gerais
}

\section{PALAVRAS-CHAVE \\ - Educação Médica. \\ - Estudante de Medicina. \\ - Sofrimento Psíquico.}

I Departamento de Clínica Médica, Universidade Federal de Minas Gerais, Belo Horizonte, Minas Gerais, Brasil.

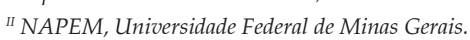

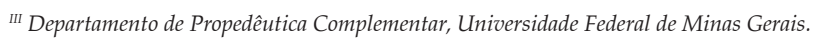




\section{KEY-WORDS}

- Medical Education

- Medical Student

- Psychological Distress.
ABSTRACT

The psychological distress experienced by university students, particularly medical students, is well-known and has long been a cause for concern. In the School of Medicine of the Universidade Federal de Minas Gerais, the School of Medicine Psychopedagogical Student Support Center (Núcleo de Apoio Psicopedagógico ao Estudante da Faculdade de Medicina - NAPEM) provides care to students who spontaneously seek help. The purpose of this study was to analyze the reasons described when completing the Napem application form by students for their seeking the assistance. A total of 273 students enrolled in the two years studied, which were both in the last decade. To preserve the identity of the students, the years analyzed were not identified. Of this group, 235 were medical students, 28 students of Speech Therapy, four undergraduate students of Technology in Radiology and six were students of other courses. The methodology used for data analysis was qualitative, with content analysis and the frequency comparison was performed using the chi-square test. The reasons to seek care written by the students in the application form were placed in five categories established by the researchers - "mood disorders," "personal problems," "personality traits," "academic issues" and others. The results showed that "mood disorders" were the most frequently reported (44.3\%), followed by "personality traits" (26.4\%) and "personal problems" (13.9\%). The "academic questions" presented the lowest frequency (5.5\%). The desire to die was described by 17 (6.2\%) students. When the medical students were compared to the students of the other courses, there was no statistically significant difference among the frequencies of the five categories $(p=0.21)$ with motives allocated in the category "mood disorders" (41.7\%) being predominantly observed. However, when considering two studied periods separately, the frequency of the category "mood disorders" for medical students was significantly higher $(p=0.0005)$ in the second year than in the first year of study. It should be emphasized that almost $50 \%$ of the students enrolled in the second year analyzed attended the centre for first two years, a significantly higher result ( $p=0.002)$ when compared to those enrolled in the first year of the study. Even recognizing the limitations regarding the analysis of the student's preliminary demand for care when seeking help, particularly when expressed in written form, it is believed that the results of this study can contribute to the organization of psychological support services. Due to the magnitude of the problem, there is a clear need to have welcome spaces for the students with activities that can receive them and listen to them when they enter college, such as mentoring programs.

Recebido em 20/2/19

Aceito em 20/5/19

\section{INTRODUÇÃO}

O sofrimento psíquico entre estudantes de Medicina e médicos é bem conhecido e estudado ${ }^{1-6}$. Na Faculdade de Medicina da Universidade Federal de Minas Gerais (FM-UFMG), a preocupação com o sofrimento psíquico do estudante não é recente. O Núcleo de Apoio Psicopedagógico ao Estudante da FM-UFMG (Napem) existe, oficialmente, desde 2004 e tem como missão a assistência ao estudante da faculdade que o procura. Além de prestar assistência psicológica, a equipe responsável pelo Napem tem buscado compreender os fatores geradores de estresse psicológico no estudante e os meios possíveis para sua prevençâo $0^{7-10}$.

$\mathrm{Na}$ dinâmica do funcionamento do Napem, o primeiro passo para o atendimento é o preenchimento, pelo aluno, de uma ficha de inscrição. Nesta ficha, além de informar dados sociodemográficos, o aluno explicita o motivo de sua demanda. Sabe-se que o motivo informado pelo estudante por ocasião de sua inscrição não corresponde, em muitas das vezes, à demanda real que ele apresenta, refletindo apenas o que ele considera que pode ser exposto para justificar sua busca por atendimento ${ }^{11,12}$. Constitui, entretanto, um indicativo do que ele identifica como necessidade de buscar ajuda. Além disso, permite saber se ele atribui seu sofrimento a problemas com o seu curso de graduação (Medicina ou outro) ou a problemas pessoais.

Para este estudo foram coletados dados das fichas dos estudantes que se inscreveram no Napem em dois diferentes anos na última década, sendo um deles anterior à implantação da Lei de Cotas na UFMG e o outro posterior a ela. A entrada por cotas mudou significativamente o perfil do estudante de Medicina, com aumento do número de estudantes provenientes de escolas públicas, bem como de estudantes provenientes do interior de Minas Gerais e de outros estados do Brasil e, ainda, de estudantes com renda familiar baixa ${ }^{13}$. Além disso, os dois anos avaliados contemplam importante mudança curricular no curso de Medicina da FM- UFMG.

Verificar o perfil do estudante e o motivo apresentado por ele para a busca de ajuda junto ao Napem em dois períodos 
distintos e identificar possíveis diferenças entre esses momentos foi o objetivo deste trabalho.

\section{METODOLOGIA}

A pesquisa foi realizada em banco de dados construído com base em fichas de inscrição para o atendimento no Napem. Foram analisadas as seguintes variáveis: curso, período, sexo, idade e motivo da demanda por atendimento.

Foram selecionados os dados de alunos que fizeram sua inscrição no Napem em dois anos-calendário, compreendidos entre 2010 e 2018. Os anos não estão explicitados, para evitar qualquer possibilidade de identificação de turmas e alunos.

Foram analisadas, no total, 273 fichas de inscrição, que abrangem alunos matriculados nos três cursos oferecidos na FM-UFMG. Os dados sociodemográficos foram tabulados para cálculo de frequência, e as comparações foram feitas empregando-se o teste do qui-quadrado. $\mathrm{O}$ motivo da inscrição foi objeto de análise de conteúdo, e, para isto, cada um dos três pesquisadores fez duas leituras de cada uma das fichas. Considerando-se os motivos predominantes constantes no relato dos alunos, os pesquisadores criaram, para efeito de análise estatística, cinco categorias para alocação desses motivos: alterações do humor (A); questões/problemas pessoais (B); características de personalidade (C); questões acadêmicas (D); outros motivos (E). A categorização dos motivos foi feita com base em discussões entre os pesquisadores, buscando-se a obtenção de consenso.

O projeto foi aprovado no Comitê de Ética e Pesquisa da UFMG (CAAE 73541817.2.0000. 5149).

\section{RESULTADOS}

Nos dois anos avaliados, 273 estudantes procuraram o Napem pela primeira vez e, portanto, preencheram a ficha de inscrição. A idade dos alunos variou de 17 a 64 anos, sendo a mediana de 23 anos. A distribuição dos estudantes por curso, sexo e período encontra-se descrita na Tabela 1. Na FM-UFMG funcionam três cursos de graduação: Medicina, que oferece 320 vagas anuais e tem um total de 1.920 alunos, distribuídos em 12 períodos; Fonoaudiologia, com 50 vagas anuais e 250 alunos, distribuídos em dez períodos; e o curso superior de Tecnologia em Radiologia, que oferece 80 vagas anuais com 320 alunos, distribuídos em oito períodos. Os cursos de Medicina e de Fonoaudiologia são diurnos, em horário integral, e o de Tecnologia em Radiologia é noturno.

O motivo apresentado pelo aluno para buscar atendimento no Napem foi uma descrição livre e não teve, inicialmente, objetivo de pesquisa. Variou, em extensão, de uma palavra a poucos parágrafos. A leitura dessas justificativas e sua análise
TABELA 1

Distribuição, por curso, sexo e período, dos

273 estudantes que demandaram atendimento no Napem nos dois anos avaliados

Número de alunos (\%)

Curso

Medicina $235(86,1)$

Fonoaudiologia $28(10,3)$

Tecnologia em Radiologia $04(1,4)$

Outros* $06(2,2)$

Sexo

Feminino $\quad 171(62,6)$

Masculino $102(37,4)$

Período em curso**

$\begin{array}{lc}1 & 38(14,0) \\ 2 & 28(10,3) \\ 3 & 20(7,4) \\ 4 & 12(4,4) \\ 5 & 26(9,6) \\ 6 & 24(8,9) \\ 7 & 32(11,8) \\ 8 & 19(7,0) \\ 9 & 26(9,6) \\ 10 & 19(7,0) \\ 11 & 13(4,8) \\ 12 & 14(5,2)\end{array}$

* Médicos residentes ou alunos de pós-graduação excepcionalmente atendidos no Napem. ** Dado não obtido de dois estudantes.

resultaram nas cinco categorias já descritas. No Quadro 1 estão os agrupamentos feitos para a categorização e o número de estudantes em cada categoria, sendo os motivos alocados na categoria distúrbios do humor os mais frequentemente relatados $(44,3 \%)$, seguidos por características de personalidade $(26,4 \%)$ e por problemas pessoais (13,9\%). Questões acadêmicas como motivo mais relevante para procurar atendimento no Napem aparece em menor número que todos os demais (5,5\%).

Entre os motivos alocados na categoria distúrbios do humor, o desejo de morrer foi relatado por 17 estudantes (14 do curso de Medicina, 2 do curso de Fonoaudiologia e 1 do curso de Tecnologia em Radiologia) e descrito como ideação ou pensamento suicida, tentativas prévias de suicídio, pensamento de morte, pensamento ruim, desejo de morrer ou pensamento sobre o desejo de estar vivo. Entre os outros motivos (letra E) estão situações não classificáveis nas categorias estabelecidas.

Considerando o grande número de estudantes do curso de Medicina na amostra, decidiu-se analisar separadamente a demanda dos estudantes desse curso tanto em relação ao total de alunos inscritos $(n=235)$ quanto em relação a cada um dos anos avaliados - 132 alunos inscritos no primeiro ano e 103 no segundo ano (Tabela 2). 


\begin{tabular}{|c|c|c|c|c|}
\hline \multicolumn{5}{|c|}{$\begin{array}{l}\text { QUADRO } 1 \\
\text { Agrupamentos de relatos dos alunos em categorias e } r\end{array}$} \\
\hline $\begin{array}{l}\text { A - Distúrbios do humor } \\
\qquad(\mathrm{n}=121)\end{array}$ & $\begin{array}{l}\text { B - Problemas pessoais } \\
\quad(\mathrm{n}=38)\end{array}$ & $\begin{array}{l}\text { C-Características de } \\
\text { personalidade }(n=72)\end{array}$ & $\begin{array}{l}D \text { - Questões } \\
\text { acadêmicas } \\
(n=15)\end{array}$ & $\begin{array}{l}\text { E- Outros motivos } \\
\quad(n=27)\end{array}$ \\
\hline $\begin{array}{l}\text { Depressão; ansiedade; } \\
\text { estresse; insônia; } \\
\text { angústia; instabilidade } \\
\text { emocional; falta de } \\
\text { motivação persistente; } \\
\text { síndrome do pânico; } \\
\text { sentimento de fracasso; } \\
\text { choro fácil, tristeza; } \\
\text { ideação suicida; } \\
\text { tentativas prévias de } \\
\text { autoextermínio }\end{array}$ & $\begin{array}{l}\text { Conflitos familiares, } \\
\text { conflitos internos, } \\
\text { dificuldade de adaptação } \\
\text { à cidade, dificuldade com } \\
\text { a saída da casa dos pais; } \\
\text { incerteza na escolha do } \\
\text { curso; divórcio dos pais, } \\
\text { doença de um dos pais; } \\
\text { transtornos alimentares; } \\
\text { luto/perda; uso de álcool; } \\
\text { uso de drogas; história } \\
\text { passada de abuso sexual }\end{array}$ & $\begin{array}{l}\text { Insegurança; timidez; } \\
\text { ser muito exigente; } \\
\text { ter dificuldade de } \\
\text { relacionamento }\end{array}$ & $\begin{array}{l}\text { Sobrecarga do curso/ } \\
\text { fadiga; dificuldades } \\
\text { com estudos e } \\
\text { rendimento acadêmico; } \\
\text { dificuldade de } \\
\text { adaptação à faculdade; } \\
\text { vontade de desistir } \\
\text { do curso; vontade de } \\
\text { trancar a matrícula no } \\
\text { curso }\end{array}$ & $\begin{array}{l}\text { Querer fazer } \\
\text { acompanhamento } \\
\text { psicológico; querer trocar } \\
\text { o atendimento que tem } \\
\text { na clínica privada pela } \\
\text { pública; interesse no } \\
\text { serviço gratuito; ter sido } \\
\text { encaminhado por colegas, } \\
\text { professores ou outros } \\
\text { profissionais }\end{array}$ \\
\hline
\end{tabular}

\begin{tabular}{|c|c|c|c|}
\hline \multicolumn{4}{|c|}{$\begin{array}{l}\text { TABELA } 2 \\
\text { Distribuição dos alunos do curso de Medicina }(\mathrm{n}=235) \text { por sexo, período e motivo da demanda de atendimento } \\
\text { (categorias), considerando-se o grupo total e de acordo com os períodos de estudo: ano } 1 \text { (132 alunos) e ano } 2 \text { (103 alunos) }\end{array}$} \\
\hline & $\begin{array}{c}\text { Total } \\
\text { N (\%) }\end{array}$ & $\begin{array}{l}\text { Ano } 1 \\
\text { N (\%) }\end{array}$ & $\begin{array}{l}\text { Ano } 2 \\
\text { N (\%) }\end{array}$ \\
\hline \multicolumn{4}{|l|}{ Sexo } \\
\hline Feminino & $97(41,3)$ & $49(62,9)$ & $48(46,6)$ \\
\hline Masculino & $138(58,7)$ & $83(37,1)$ & $55(53,4)$ \\
\hline \multicolumn{4}{|l|}{ Período do curso } \\
\hline 1 & $36(15,3)$ & $12(9,1)$ & $24(23,3)$ \\
\hline 2 & $28(11,9)$ & $14(10,6)$ & $14(13,6)$ \\
\hline 3 & $15(6,4)$ & $07(5,3)$ & $08(7,8)$ \\
\hline 4 & $09(3,8)$ & $05(3,8)$ & $04(3,9)$ \\
\hline 5 & $20(8,5)$ & $11(8,3)$ & $09(8,7)$ \\
\hline 6 & $17(7,2)$ & $10(7,6)$ & $07(6,8)$ \\
\hline 7 & $25(10,6)$ & $17(12,9)$ & $08(7,8)$ \\
\hline 8 & $17(7,2)$ & $10(7,6)$ & $07(6,8)$ \\
\hline 9 & $23(9,9)$ & $16(12,1)$ & $07(6,8)$ \\
\hline 10 & $18(7,7)$ & $09(6,8)$ & $09(8,7)$ \\
\hline 11 & $13(5,5)$ & $10(7,6)$ & $03(2,9)$ \\
\hline 12 & $14(6,0)$ & $11(8,3)$ & $03(2,9)$ \\
\hline \multicolumn{4}{|l|}{ Categorias* } \\
\hline A & $98(41,7)$ & $42(31,8)$ & $56(54,4)$ \\
\hline B & $34(14,5)$ & $25(18,9)$ & $09(8,7)$ \\
\hline $\mathrm{C}$ & $64(27,2)$ & $33(25,0)$ & $31(30,0)$ \\
\hline $\mathrm{D}$ & $13(5,5)$ & $12(9,1)$ & $01(0,1)$ \\
\hline E & $26(11,1)$ & $20(15,2)$ & $06(5,8)$ \\
\hline
\end{tabular}

\footnotetext{
${ }^{*} A=$ distúrbios do humor; $B=$ questões e problemas pessoais; $C=$ características de personalidade; $D=$ questões acadêmicas; $E=$ outros motivos.
} 
Não foi observada diferença estatisticamente significativa na frequência das categorias $A, B, C, D$ e E $(p=0,21)$ quando comparados os estudantes do curso de Medicina com os estudantes dos demais cursos.

Por outro lado, entre os estudantes de Medicina, a frequência de motivos alocados na categoria distúrbios de humor foi significativamente maior ( $p=0,0005)$ entre os inscritos no segundo ano de estudo do que entre os inscritos no primeiro ano. Além disso, o número de alunos que cursava os períodos iniciais (primeiro ao quarto período) também foi significativamente maior $(p=0,002)$ entre os inscritos no segundo ano do estudo quando comparados aos inscritos no primeiro ano.

\section{DISCUSSÃO}

Como considerado anteriormente, o motivo explicitado para a busca de acompanhamento psicoterapêutico, mesmo quando expresso para o profissional, pode não corresponder à realidade. Entretanto, os dados obtidos permitem conhecer um pouco sobre a demanda de um serviço de apoio psicopedagógico numa faculdade de Medicina disponível para a procura espontânea pelos estudantes.

Comparativamente ao total de alunos de cada curso, o percentual de estudantes do curso de Tecnologia em Radiologia que procurou o Napem foi inexpressivo. Este dado pode estar ligado ao fato de esse ser um curso noturno, e o Napem funcionar no período diurno, o que dificulta o acesso de alunos, principalmente daqueles que trabalham durante o dia.

O principal motivo de busca por atendimento, tanto para o conjunto total de estudantes quanto para os estudantes do curso de Medicina, foi aquele classificado como distúrbio de humor, que incluiu as palavras ansiedade, depressão e sintomas que pudessem sugerir esses transtornos. Em análise de casuística de 21 anos de assistência psicológica aos estudantes de Medicina da Universidade de São Paulo, Milan e Arruda ${ }^{14}$ relataram que os distúrbios de humor foram o principal motivo de consulta. Embora os problemas acadêmicos, de forma explícita, tenham sido pouco relatados, esses mesmos autores chamam atenção para as exigências do curso, associadas às características de personalidade, que desencadeiam quadros de ansiedade e depressão.

As características de personalidade, como exigência excessiva, insegurança, dificuldades de relacionamento e timidez, dificultam o enfrentamento dos desafios exigidos para lidar com o ser humano e seu adoecimento. Em estudo realizado com alunos do curso de Medicina em universidade alemã, foi observado que se sentir seguro era um fator de proteção contra o estresse ${ }^{15}$. Em outro estudo, a menor flexibilidade na resolução de problemas foi descrita como um dos fatores de risco para o suicídio ${ }^{16}$. O fato de $6,2 \%$ dos estudantes atendidos terem feito menção ao suicídio é preocupante ao chamar atenção para a gravidade do adoecimento mental entre aqueles que demandaram assistência.

Dificuldades pessoais foram causa importante para a busca de ajuda. Aliadas à imaturidade emocional e à evitação de problemas, poderiam favorecer o adoecimento mental ${ }^{15}$. Vale ressaltar que a mediana de idade dos estudantes analisados foi de 23 anos, ou seja, são, em sua maioria, pessoas jovens, ainda em fase de amadurecimento emocional.

Quando se analisaram apenas os estudantes de Medicina, separando-se os dois anos avaliados, alguns aspectos se destacaram. Os distúrbios de humor se mantêm como os mais prevalentes e se mostram em percentual mais alto no segundo ano estudado. As características de personalidade permanecem como a segunda demanda mais importante. É sabido que o vestibular para o curso de medicina na UFMG é um dos mais concorridos do País ${ }^{13}$, selecionando pessoas competitivas e com elevado potencial intelectual e conhecimento teórico. Entretanto, a vida e o próprio curso médico exigem mais do que isso: a falta de habilidades sociais, dificuldades para lidar com frustrações e incerteza quanto à escolha da profissão são motivos de sofrimento ${ }^{17}$. Um fator agravante observado recentemente, após a implantação do sistema de seleção pelo Sisu e já relatado para outros cursos de graduação, é a escolha do curso de Medicina feita em função da nota obtida pelo estudante no Enem e não pelo desejo de se tornar médico. Se fazer uma opção profissional aos 17 ou 18 anos de idade, época do término do ensino médio, não é uma tarefa simples, a escolha da profissão em função da nota obtida no sistema de seleção tende a agravar o problema. Além disso, pelo prestígio social que essa profissão ainda detém, não é fácil para o aluno abandonar o curso de Medicina caso ele conclua que não era essa a profissão que gostaria de exercer.

Chama atenção o elevado número de alunos dos quatro períodos iniciais do curso no segundo ano estudado, chegando a quase $50 \%$ do total de estudantes que se inscreveu neste ano. Embora a literatura relate um nível maior de estresse nos anos iniciais do curso médico ${ }^{18}$, dois fatores podem ter influenciado o aumento de demanda por estudantes dos períodos iniciais. O curso de Medicina da UFMG passou por uma reforma curricular entre o primeiro e o segundo ano do estudo, e o ciclo básico apresentou dificuldades para os alunos, como excesso de conteúdo em relação à carga horária e excesso de atividades avaliativas, o que vem sendo objeto de avaliação e ajuste. Não menos importante foi a adesão da UFMG ao processo seletivo do Sisu e à Lei de Cotas, com aumento do número de estudantes do interior de Minas Gerais e de outros estados e 
de estudantes com renda familiar mais baixa ${ }^{13}$. A vulnerabilidade social em um curso tradicionalmente elitizado e a falta de suporte sociofamiliar têm consequências na saúde mental e, muitas vezes, dificultam a adesão do aluno ao acompanhamento proposto. Esses fatores têm sido observados, na prática, pelos profissionais que atendem os estudantes no Napem.

O predomínio de homens que buscam ajuda no curso de Medicina chama atenção e parece contrariar a literatura, que relata não só a existência de maior número de mulheres ${ }^{14}$ nessa situação, como também maiores níveis de estresse entre elas $^{18}$. Os dados não permitem uma discussão mais profunda, pois, mesmo conhecendo o número de estudantes homens e mulheres matriculados na faculdade em cada um dos anos, como a análise é da casuística de um serviço que atende demanda espontânea, considerou-se que não seria possível nenhuma conclusão a esse respeito.

Finalizando, considera-se que o principal motivo alegado pelos alunos para a procura de um serviço de apoio foram os distúrbios do humor, em gravidade variável, incluindo ideias de morte. Ressalta-se a importância de acolhimento do aluno o mais brevemente possível para detecção e encaminhamento adequado daqueles com maior gravidade.

$\mathrm{O}$ aumento do número de estudantes que procuram o Napem já no início do curso demanda, da Faculdade de Medicina, maior conhecimento do estudante que está chegando, além de análise cuidadosa de aspectos do próprio curso. Atividades de tutoria parecem ter papel cada vez mais relevante nesses dois aspectos e devem estar diretamente relacionadas com os serviços de apoio ao estudante.

\section{AGRADECIMENTOS}

Os autores agradecem a todos os membros do NAPEM pelo apoio na realização desse trabalho.

\section{REFERÊNCIAS}

1. Millan LR, Rossi E, De Marco OLN. A psicopatología do estudante de medicina. In: Millan LR, De Marco OLN, Rossi E, Arruda PCV. O universo psicológico do futuro médico: vocação, vicissitudes e perspectivas. São Paulo: Casa do Psicólogo; 1999. p.85-101.

2. Bellodi PL, Martins MA. A formação médica e o aluno. In: Bellodi PL. Tutoria: mentoring na formação médica. São Paulo: Casa do Psicólogo; 2005. p.121-5.

3. Ribeiro MGS. Sofrimento psíquico entre estudantes de medicina da UFMG: uma contribuição da Assessoria de Escuta Acadêmica. Belo Horizonte, 2014. Mestrado [Dissertação] - Faculdade de Medicina da Universidade Federal de Minas Gerais.
4. Silva AG, Cerqueira ATAR, Lima MCP. Apoio social e transtorno mental comum entre estudantes de medicina. RevBrasEpidemiol 2014;17(1)229-42.

5. Silva FB, Mascia AR, Lucchese AC, De Marco MA, Martins MCFN, Martins LAN. Atitudes frente a fontes de tensão do curso médico: um estudo exploratório com alunos do segundo e do sexto ano. RevBrasEducMéd 2009;33(2)230-9.

6. Lima MCP, Domingues MS, Cerqueira ATAR. Prevalência e fatores de risco para transtornos mentais comuns entre estudantes de medicina. Rev Saúde Pública 2006;40(6)103541.

7. Aquino MT. Prevalência de transtornos mentais entre estudantes de medicina da Universidade Federal de Minas Gerais. Belo Horizonte, 2012. Mestrado [Dissertação]. Faculdade de Medicina da Universidade Federal de Minas Gerais.

8. Carvalho MB, Ribeiro MMF, Silva LD, Shimomura FM. A composição do curriculum vitae entre estudantes de medicina e seus condicionantes. RevBrasEducMéd2013;37(4)483-91.

9. Corrêa RC, Gonçalves RCB, Oliveira LS, Silva VCM, Ribeiro MMF. Medicina como nova graduação: motivações, dificuldades, expectativas.RevBrasEducMéd 2016;40(2)22633.

10. Silva MAM. A percepção dos professores do curso de Medicina da Universidade Federal de Minas Gerais em relação ao sofrimento psíquico de seus alunos. Belo Horizonte; 2016.Mestrado [Dissertação] - Faculdade de Medicina da Universidade Federal de Minas Gerais.

11. Rocha F. Algumas considerações sobre as entrevistas preliminares, demanda e início de análise. Percurso 1991;3(5/6)15-21.

12. Entrevista com Fernando Rocha. ALTER - Revista de Estudos Psicanalíticos 2012; 30 (1)123-130.

13. Nonato BF. Lei de Cotas e Sisu:análise dos processos de escolha dos cursos superiores e do perfil dos estudantes da UFMG antes e após as mudanças na forma de acesso às Instituições Federais. Belo Horizonte; 2018. Tese [Doutorado] - Faculdade de Educação da Universidade Federal de Minas Gerais.

14. Millan LR, Arruda PCV. Assistência psicológica ao estudante de medicina: 21 anos de experiência. Rev Assoc Med Bras 2008;54(1)90-4.

15. van Dijk I, Lucassen PLBJ, van Weel C, Speckens AEM. A cross-sectional examination of psychological distress, positive mental health and their predictors in medical students in their clinical clerkships. BMC Medical Education2017;17:219-29. 
16. Keller M, Guevara S. Flexibilidade na resolução de problemas em tentadores de suicídio. J BrasPsiquiatr 2005;54(2)128-36.

17. Santa ND,Cantilino A.Suicídio entre Médicos e Estudantes de Medicina: Revisão de Literatura. RevBrasEducMed [online]. 2016. 40(4) [capturado em 13 fev. 2019];772-780. Disponível em: http://www.scielo.br/scielo.php?pid= S010055022016000400772 \&script=sci_abstract\&tlng=pt

18. Roberto A, Almeida A. A saúde mental de estudantes de medicina: estudo exploratório na Universidade da Beira Interior. Acta MedPort2011;24(S2)279-86.

\section{CONTRIBUIÇÃO DOS AUTORES}

Maria Mônica Freitas Ribeiro participou da idealização do projeto, da pesquisa bibliográfica, da orientação metodológica, da análise dos dados, e da redação do artigo. Jordan Diego Costa Melo participou da idealização do projeto, da pesquisa bibliográfica e da obtenção e análise dos dados. Andreia Maria Camargos Rocha participou da orientação metodológica, da análise dos dados e da redação do artigo.

\section{CONFLITO DE INTERESSES}

Declaramos não haver conflito de interesses

\section{ENDEREÇO PARA CORRESPONDÊNCIA}

Maria Mônica Freitas Ribeiro

Departamento de Clínica Médica, Faculdade de Medicina/ UFMG

Av. Alfredo Balena 190

30130-100, Belo Horizonte, MG, Brasil

mmonica@medicina.ufmg.br 